Hot Topic

\title{
Toll like receptors and pancreatic diseases: From a pathogenetic mechanism to a therapeutic target
}

\author{
Matteo Santoni ${ }^{a}$, Kalliopi Andrikou ${ }^{a}$, Valeria Sotte ${ }^{a}$, Alessandro Bittoni ${ }^{a}$, Andrea Lanese ${ }^{a}$, Chiara Pellei ${ }^{a}$, \\ Francesco Piva ${ }^{\mathrm{b}}$, Alessandro Conti ${ }^{\mathrm{a}}$, Massimo Nabissi ${ }^{\mathrm{c}}$, Giorgio Santoni ${ }^{\mathrm{c}}$, Stefano Cascinu ${ }^{\mathrm{a}, *}$ \\ a Clinica di Oncologia Medica, AOU “Ospedali Riuniti”, Università Politecnica delle Marche, via Conca 71, 60126 Ancona, Italy \\ ${ }^{\mathrm{b}}$ Department of Specialistic Clinical and Odontostomatological Sciences, Polytechnic University of Marche, Ancona 60131, Italy \\ 'School of Pharmacy, Experimental Medicine Section, University of Camerino, via Madonna delle Carceri, 62032 Camerino, Italy
}

\section{A R T I C L E I N F O}

\section{Article history:}

Received 17 February 2015

Received in revised form 5 April 2015

Accepted 6 April 2015

Available online $\mathrm{xxxx}$

\section{Keywords:}

Diabetes

Inflammation

Pancreatic Ductal Adenocarcinoma

Pancreatitis

Toll like receptors

\begin{abstract}
A B S T R A C T
Toll-like receptors (TLRs) mediate interactions between environmental stimuli and innate immunity. TLRs play a major role in the development of numerous pancreatic diseases, making these molecules attractive as potential therapeutic targets. TLR2, TLR7 and TLR9 are involved in the initiation of type 1 diabetes mellitus (T1DM), whereas TLR2 and TLR4 play a major role in the onset of type 2 diabetes mellitus (T2DM). Furthermore, TLRs cause derangements in several tumor suppressor proteins (such as p16, p21, p27, p53 and pRb), induce STAT3 activation and promote epithelial-mesenchymal transition as well as oncogene-induced senescence. In this review we will focus on the contribution of TLRs in pancreatic disease including cancer and we describe recent progress in TLR-modulation for the treatment of these patients.
\end{abstract}

(c) 2015 Elsevier Ltd. All rights reserved.

\section{Introduction}

Activation of innate immunity is achieved through the stimulation of pattern recognition receptors (PRRs). Among them, Toll-like receptors (TLRs) were the first group to be identified. They can be activated by a panel of pathogen-associated molecular patterns (PAMPs) [1,2] and alarmins [3] endogenous molecules released by activated or necrotic cells in response to stress or tissue damage into the extracellular compartment [4].

Another feature of PPRs is their capability to recognize self-molecular patterns originated from damaged cells, named Damage Associated Molecular Patterns (DAMPs).

TLRs are single-pass transmembrane proteins with an intracellular C-terminal tail known as the Toll/IL-1 receptor (TIR) and an extracellular N-terminal that contains leucine-rich repeats (LRRs). TLR ligation leads to activation of two major intracellular signaling pathways. All TLRs, except TLR3, can activate a Myeloid differentiation primary response protein 88 (MyD88)-dependent pathway (Fig. 1). This pathway involves IL-1R-associated kinases (IRAK),

\footnotetext{
* Corresponding author. Tel.: +39 0715964169; fax: +39 0715964269.

E-mail addresses: mattymo@alice.it (M. Santoni), k.andrikou@hotmail.com (K. Andrikou), valeria.sotte@mail.com (V. Sotte), alebitto@tiscali.it (A. Bittoni), andrealanese@gmail.com (A. Lanese), chiarapellei@libero.it (C. Pellei), fpiva@ univpm.it (F. Piva), alessandro83conti@gmail.com (A. Conti), massimo.nabissi@ unicam.it (M. Nabissi), giorgio.santoni@unicam.it (G. Santoni), s.cascinu@univpm.it (S. Cascinu).
}

tumor necrosis factor (TNF) receptor-associated factor 6 (TRAF-6) and mitogen-activated kinases and leads to the transcription of pro-inflammatory genes through the activation of nuclear factor $\kappa \beta(\mathrm{NF} \kappa \mathrm{B})$ and/or the activation of activating protein $1[5,6]$. Furthermore, TLR3 and TLR4 can activate the TIRAP inducing interferon $\beta$ (TRIF) pathway, leading to the synthesis of interferon $\alpha / \beta$ $($ IFN- $\alpha / \beta)[5]$.

At present, twelve TLRs have been identified in mice (TLR1TLR9 and TLR11-TLR13) and ten in human (TLR1-TLR10) [3]. Most TLRs are on the cell surface, except for TLR3, $-7,-8$, and -9 , mainly present in the endosomes [2]. A further classification divides TLRs based on the type of recognized PAMPs: TLR1, TLR2, TLR4 and TLR6 detect lipids, whereas TLR5 and TLR 10 recognize proteins and TLR3, TLR7, TLR8 and TLR9 detect nucleic acids [5]. The list of PAMPs and alarmins recognized by human TLRs is shown in Table 1.

The involvement of TLRs in the pathophysiology of several diseases has become a major research field [6,7]. This review summarizes the role of TLRs in the pathogenesis of inflammatory related pancreatic disease as well of pancreatic cancer, highlighting their potential use as future therapeutic targets.

\section{Methods}

Data for this review were identified from the Pubmed database, using the subsequent MeSH (Medical Subject Heading) terms: 


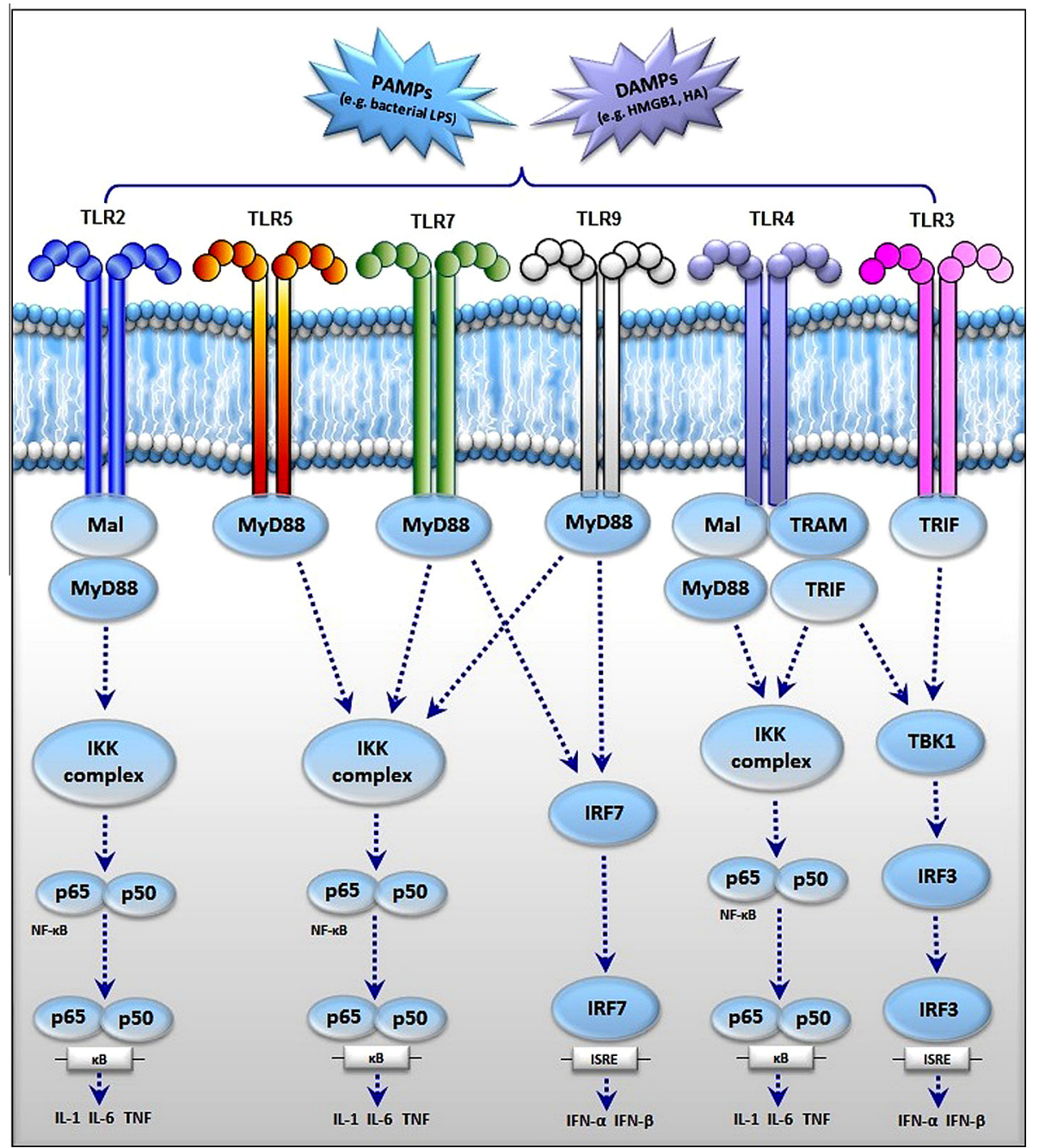

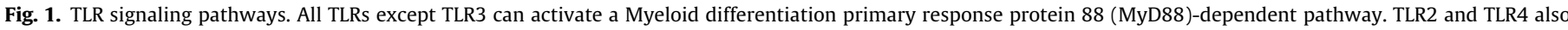

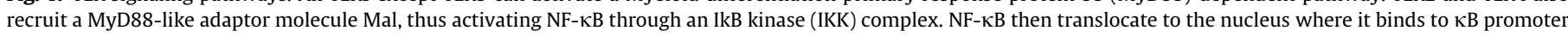

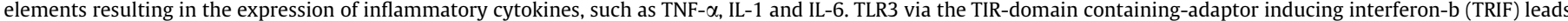

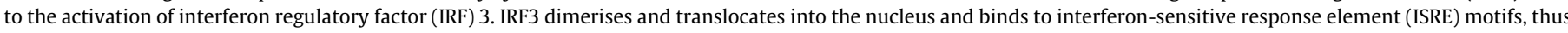

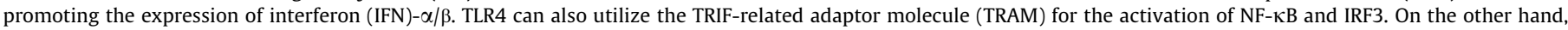
TLR7 and TLR9 can also activate IRF3 related molecules such as IRF7, leading to the expression of IFN- $\alpha / \beta$.

Table 1

Ligand recognition by Toll-like receptors.

\begin{tabular}{|c|c|c|c|}
\hline TLRs & Localization & PAMP & Origin of PAMP \\
\hline TLR1 & Plasma membrane & Triacyl lipopeptides & N. meningitidis triacyl lipopeptides bacteria, mycobacteria \\
\hline TLR2 & Plasma membrane & $\begin{array}{l}\text { Glycoinositolphospholipids, glycolipids, } \\
\text { haemagglutinin, lipoarabinomannan, lipoprotein/ } \\
\text { LIPOPEPTIDES, lipoteichoic acid, zymosan, } \\
\text { peptidoglycan, phenol-soluble modulin, porins }\end{array}$ & $\begin{array}{l}\text { Trypanosoma cruzi, Treponema maltophilum, virus, mycobacteria, } \\
\text { various pathogens, gram-positive bacteria, gram-positive bacteria, } \\
\text { S. epidermidis, neisseria, fungi }\end{array}$ \\
\hline TLR3 & Endosome & Double-stranded RNA & Virus \\
\hline TLR4 & Plasma membrane & $\begin{array}{l}\text { Envelope protein, fusion protein, heat-shock } \\
\text { protein } 60 \text {, lipopolysaccharide, taxol }\end{array}$ & $\begin{array}{l}\text { Mouse-mammary tumor virus, respiratory syncytial virus, } \\
\text { Chlamydia pneumoniae, gram-negative bacteria, plants, }\end{array}$ \\
\hline TLR5 & Plasma membrane & Flagellin & Bacteria \\
\hline TLR6 & Plasma membrane & Diacyl lipopeptides, zymosan, lipoteichoic acid & Mycoplasma, gram-positive bacteria, fungi \\
\hline TLR7 & Endosome & Single-stranded RNA & Virus \\
\hline TLR8 & Endosome & Single-stranded RNA & Virus \\
\hline TLR9 & Endosome & DNA ( $\mathrm{CpG}$ ), haemozoin & Bacteria, virus, Plasmodium spp., Rhodnius spp., Schistosoma spp. \\
\hline TLR10 & Endosome & Not determined & Not determined \\
\hline
\end{tabular}

“Inflammation”, “Immune Response" “TLR”, “Toll-like receptor", each combined with "Cancer", "Diabetes", "Pancreatitis", "Pancreatic cancer", "Pancreatic Ductal Adenocarcinoma”, "Sterile Inflammation", and "Systemic Inflammatory Response Syndrome
(SIRS)". A further search was done of related articles and references from relevant papers. The ongoing trials were searched out on the official website www.clincaltrials.gov, with the last search on April 2014. No language was restricted. The final reference list was 
generated on the basis of originality and relevance to the broad scope of this review.

\section{Role of TLRs in acute pancreatitis}

Acute pancreatitis (AP) is characterized by early activation of intracellular proteases followed by acinar cell death and inflammation. Mild acute pancreatitis (MAP), also known as edematous acute pancreatitis represents $80 \%$ of AP. While patients with MAP commonly recover without complication since MAP is a self-limited disease, in severe acute pancreatitis (SAP) (20\%) there are frequent local and extrapancreatic complications inducing systemic inflammatory response syndrome (SIRS) and sequential multiple organ disfunction syndrome (MODS) [8].

Acinar and fat cells are the early cells damaged in AP, causing disregulation in basolateral secretion and enhanced ductal permeability. This event leads to early plasmatic increase of clinical markers of pancreatic injury, such as lipase and amylase [9]. The intracellular protective mechanisms that prevent enzymes activation include synthesis of trypsin as inactive enzyme trypsinogen, autolysis of activated trypsin, enzyme compartmentalization, synthesis of specific trypsin inhibitors such as serine protease inhibitor Kazal type 1 (SPINK1), and lowering of intracellular ionized $\mathrm{Ca}^{2+}$ concentrations.

A wider enzymatic activation, involving elastase phospholipase A2, complement and kinin pathways, occurs after tripsinogen activation into trypsin in acinar cells. These events cause gland autodigestion, local inflammation and lastly the release of intracellular contents from necrotic cells. The transendothelial migration of leucocytes amplifies the tissue damage, due to the release of leucocyte harmful enzymes, the generation of oxygen-derived free radicals and the increase of pancreas hypoxia caused by vessel damages, amplifying the pro-inflammatory condition [8].

In health conditions, DAMPs are sequestered inside the cell, but after tissues injury, they are released in the extracellular space becoming available to cell surface PRRs. Since DAMPs engage the same PRRs as microbial agents, their inflammatory state has been indicated as "sterile inflammation" (Fig. 2) [9]. This status involves TLRs (TLR4 or TLR9) and the signaling launched by the membrane purigenic receptor P2X and intracellular NOD-like receptors (NLR), which leads to the activation of Caspase 1, a cytosolic cystein protease that regulates the conversion of pro-cytokines into mature forms [9]. A number of DAMPS (such as high-mobility group box protein 1 (HMGB1), adenosine triphosphate and heat shock protein

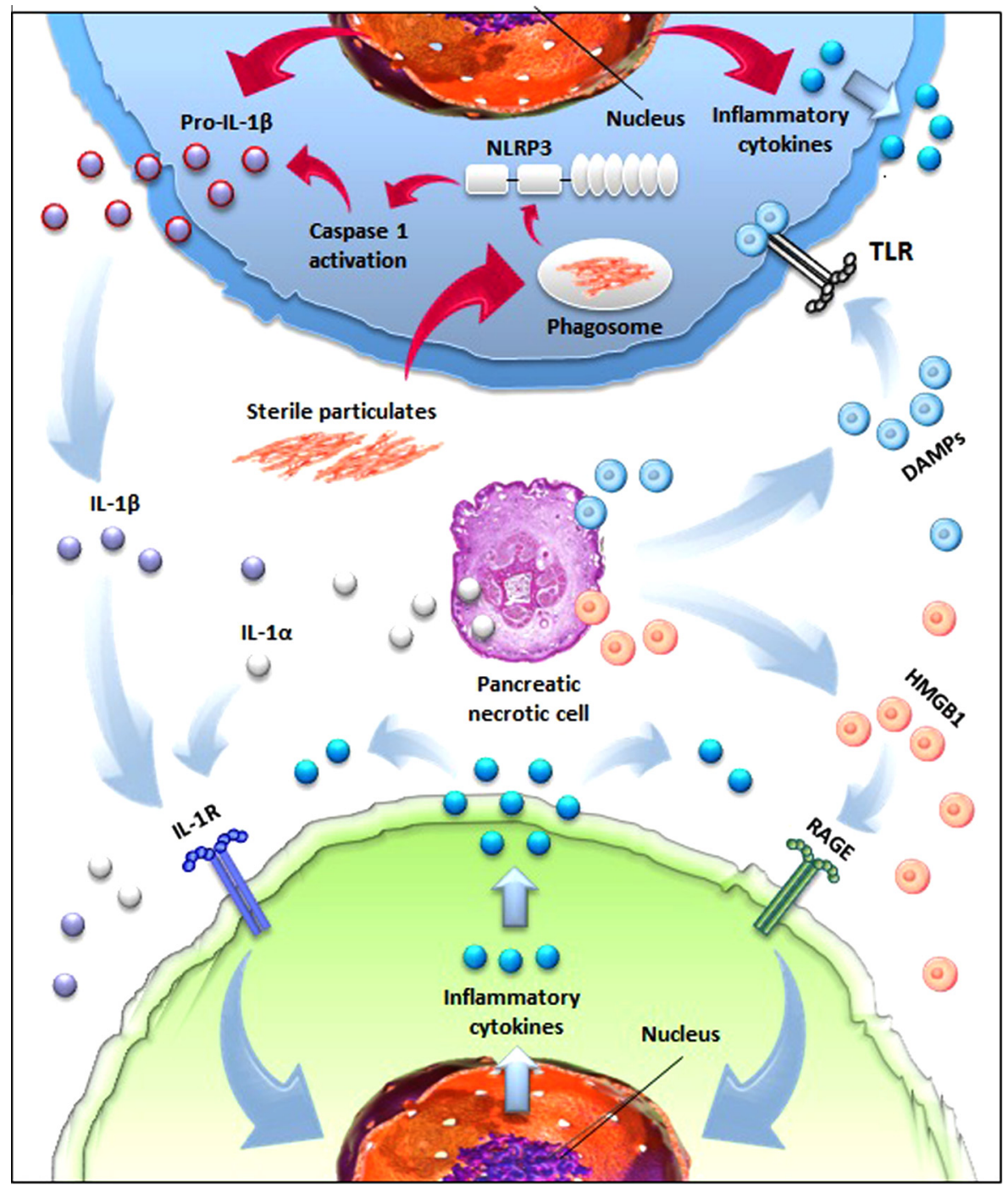

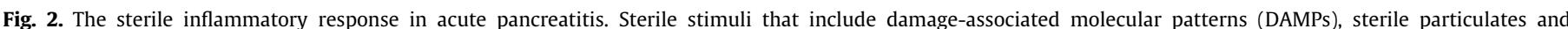

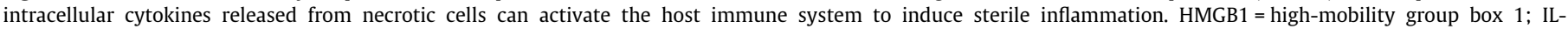

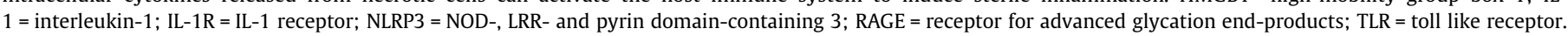


70) have been shown to play a role in experimental pancreatitis. HMGB1 is released by injured acinar cells and acts as a proinflammatory cytokine with neoangiogenetic and chemotactic activity. It affects cell proliferation and regeneration of damaged tissues stimulating autophagy [10]. Furthermore, HMGB1 activates sterile inflammation through TLR, especially TLR4. TLR4 is expressed not only on immune system cells, but also in pancreatic ductal and endothelial cells or in tissue macrophages (thus called DAMP-sensing cells). Notably, TLR4 is not expressed in acinar cells [9]. In mice with caerulein-induced pancreatitis (CIP), TLR4 deficiency is associated with a delayed development of pancreatitis [11]. Similarly, other reports show that acinar cell necrosis, edema and hemorrhage induced by taurocholate are significantly decreased in TLR4-deficient mice [12].

TLR9 is expressed in immune cells, pancreatic ductal and endothelial cells, but not in acinar cells. TLR9 recognizes microbial nucleic acid, specifically CpG motifs (unmethylated cytosine-phosphate-guanine $(\mathrm{CpG})$ dinucleotides in DNA sequences). Host genomic DNA released by necrotic acinar cells is elevated in serum as very early AP event and is recognized as a DAMP by TLR9 promoting an immune activation of sterile inflammation [9]. In addition, TLR9 induced by HMGB1 promotes the formation of CpG-DNA-TLR9 complex. HMGB1 interacts and preassociates with TLR9 in the endoplasmic reticulum-Golgi intermediate compartment (ERGIC), and hastens TLR9 redistribution to early endosomes in response to CpG-DNA. Loss of HMGB1 leads to a defect in response to CpG-DNA in terms of IL-6, IL-12, TNF- $\alpha$, and inducible nitric-oxide synthase expression [13]. Interestingly, the genetic deletion of TLR9 in pancreatitis leads to reduced pancreatic edema, inflammation, and pro-IL-1 $\beta$ expression [14].

\section{Systemic inflammatory response syndrome (SIRS) in AP: role of TLRS}

SIRS is a clinical condition characterized by a specific physiological alteration of body temperature, white blood cell count, heart and respiratory rates. SIRS and sepsis may be differentiated by the presence or absence of a focus of infection. AP represents one of the most common non-infectious causes of SIRS [15].

In AP, SIRS leads to distant organ damage, hypotension or hypoperfusion and MODS, which is the primary cause of morbidity and mortality in this condition.

TLRs have been shown to play a major role in the development of SIRS. However, the recognition of PAMP by TLRs is not able to explain the etiology of SIRS or the pathogenesis of such "sterile conditions" as ischemia and atherosclerosis, where no infection can be found. Zhai et al. reported that ischemic injury to the liver does not occur in mutant mice hosting a non-functional TLR4, and that the activation of TLR4 is not mediated by lipopolysaccharide (LPS), the archetypic agonist for TLR4 [16]. In addition, the ability of heparin sulfate and pancreatic elastase to induce SIRS is greatly diminished in TLR4-mutant mice [17].

A central role in the initiation, maintaining and progression of AP to SIRS is played by pro-inflammatory mediators such as tumor necrosis factor (TNF) and IL-1, IL-6, IL-8, IL-10, PAF, C5a and ICAM-1 [18]. Their secretion and expression is closely regulated by the activation of gene promoters through NF- $\kappa B$, which is the ending point of TLRs signaling pathway [19]. Moreover, TLR2 and TLR4 seem to be involved in lung and liver injuries as complications of AP [20].

\section{Therapeutic perspectives}

Despite a lack of studies specifically evaluating the role of TLR-modulation in the clinical setting of AP, there is a growing body of evidence that both the TLRs and downstream TLR signaling pathways may be promising therapeutic targets (Table 2).

Several agents have been proposed to modulate TLR4 activities. Eritoran, is a structural inhibitor of the lipid A portion of LPS, has been shown to inhibit TLR4 [21]. On the other hand, Resatorvid (TAK 242) is a selective inhibitor of signaling from the intracellular domain of TLR4 [22]. However, a non-significant reduction in mortality rate in patients with severe sepsis has been reported $[23,24]$. Therefore, the potential use of TLR4 antagonists in patients with AP or SIRS is still controversial.

TLR9 is important DAMP receptor upstream of inflammasome activation, and its antagonism could provide a new therapeutic strategy for treating AP. In 2011, Hoque et al. revealed that components of the inflammasome (apoptosis-associated speck-like protein containing a caspase recruitment domain [ASC], NLRP3, caspase-1), as well as TLR9, were essential for CIP development in wild-type mice and mice deficient in inflammasome components. Pretreatment with IRS954 (TLR9 antagonist) reduced pancreatic edema, inflammatory infiltrate, pancreatic necrosis and lung inflammation in taurolithocholic acid 3-sulfate-induced AP [14].

The inhibition of HMBG1, by using of blocking antibodies, decreases pancreatic injury and lung inflammation in an experimental model of severe AP [25]. Similarly, pharmacologic antagonism of both TLR9 and TLR7 decreases acinar cell necrosis and lung injury in an experimental model of severe AP [14].

Lysosomal acidification is required for endosomal TLR-mediated immune responses. Chloroquine, through the inhibition of endosomal acidification, has been shown to reduce pancreatic injury and mortality in an experimental AP model [26].

Furthermore, the recombinant IL-1 receptor antagonist has been shown to decrease pancreatic injury and inflammation [27].

Peroxisome proliferator-activated receptor- $\alpha$ (PPAR- $\alpha$ ) has attracted considerable attention for its anti-inflammatory properties. It has been reported that the administration of the PPAR- $\alpha$

Table 2

Selection of agents under evaluation in the treatment of AP. IL: interleukin; MIF: migration inhibitory protein; TLR: Toll-like receptor; TNF- $\alpha=$ tumor necrosis factor- $\alpha$.

\begin{tabular}{|c|c|c|}
\hline Agent & Description & Activity in AP \\
\hline WY14643 & Peroxisome proliferator-activated receptor- $\alpha$ agonist & $\begin{array}{l}\text { Markedly decreases TLR2 and TLR } 4 \text { mRNA and proteins, along with } \\
\text { IL-6, ICAM- } 1 \text { and TNF- } \alpha \text { mRNA levels }\end{array}$ \\
\hline Chloroquine & Weak base that accumulates in the lysosomes and increases their $\mathrm{pH}$ & Inhibit endosomal acidification, required for some TLR activation \\
\hline L-Arg & 2-Amino-5-guanidinopentanoic acid (amino acid) & $\begin{array}{l}\text { Down-regulates TLR2 and TLR4 and stimulates the production of nitric } \\
\text { oxide, meliorating lung and liver damages }\end{array}$ \\
\hline IRS954 & TLR9 antagonist & Reduces pancreatic edema, inflammatory infiltrate, and apoptosis \\
\hline Emodin & 1,3,8-trihydroxy-6-methylanthraquinone & Suppress TLR4 up-regulation \\
\hline Eritoran & Structural inhibitor of the lipid A portion of LPS & Inhibits TLR4 activity \\
\hline TAK 242 & Cyclohexene derivative & Inhibits TLR4 signaling \\
\hline Danshen & $\begin{array}{l}\text { Dried root and rhizome of the medicinal plant } \\
\text { Salvia miltiorrhiza Bunge (Labiatae) }\end{array}$ & $\begin{array}{l}\text { Inhibits the binding of LPS to TLR4, reducing bacterial translocation } \\
\text { and liver injury }\end{array}$ \\
\hline Anti-MIF antibody & $20 \mu \mathrm{g} /$ animal & Suppress the AP-induced elevation of TLR- 4 pulmonary expression \\
\hline Baicalin & Flavonoid, prolyl endopeptidase inhibitor & Suppress TLR4 up-regulation \\
\hline
\end{tabular}


agonist WY14643 in rats with CIP reduced amylase, lipase, myeloperoxidase activity, as well as IL-6, and ICAM-1 levels. Moreover, the TLR2 and TLR4 mRNA and proteins were markedly decreased by WY14643, along with IL-6, ICAM-1, and TNF- $\alpha$ mRNA levels, suggesting for a potential use of WY14643 in AP attenuation [28].

\section{Role of TLRs in diabetes mellitus and its complications}

Diabetes is among the leading causes of death and disability affecting more than 348 million people worldwide [29]. Its incidence is rapidly increasing, and by 2030 , this number is estimated to almost double [30]. A growing body of evidence has indicated that diabetes and its complications underlie a proinflammatory state characterized by elevated plasma C-reactive protein, cytokines (IL-1, TNF- $\alpha$, IL-6), chemokines, adhesion molecules, and monocyte activity.

Furthermore, the activation of the innate immune system via TLRs, TLR2 and TLR4 in particular, seems to play a key role in the pathogenesis of both type 1 (T1DM) and type 2 diabetes mellitus (T2DM).

While apoptosis of pancreatic $\beta$-cells, after a long sequence of autoimmune processes, appears to be the last step in the development of T1DM, the initial event in the pathogenesis of T1DM has not been cleared, although several authors proposed the sensing of DAMP from $\beta$-cells by TLR2 on dendritic cells (DCs) as the first event. An increase in receptors and mRNA expression of TLR2 and TLR4 in monocytes was reported from patients with T1DM, without vascular complications, compared to healthy controls [31], as well as an upregulation of downstream targets of TLR signaling including MyD88, TRIF, phosphorylated IL-1 receptor-associated kinase, and NF- $\kappa B$. In addition, elevated TRL2 and TLR4 expressions are significantly associated with glycemic control and advanced glycation end products. Knockdown of both TLR2 and TLR4 resulted in a 76\% decrease in high glucose-induced NF- $\kappa B$ response, suggesting an addictive effect [32-34]. Kim et al. found that diabetogenic T cell priming and the development of autoimmune diabetes were significantly inhibited in TLR2-null NOD mice. These data suggest that TLR2 blockade could be used in the treatment of autoimmune diabetes [34]. Furthermore, Mohammad and colleagues showed that TLR2 and TLR4 expression was increased in T1DM non-obese mice and correlated with NF- $\mathrm{BB}$ activation in response to LPS. These results were also confirmed in a study in streptozotocin-induced diabetic mouse models [35].

TLRs seem to be involved even in the pathogenesis of T2DM. An increased mRNA expression of TLR2 and TLR4 in peripheral monocytes and increased TLR2 expression only in subcutaneous adipose tissue in T2DM was observed [36]. Moreover, an excess of glucose and free fatty acid (FFA) induced elevated TLR2 and TLR4 mRNA and protein expression in monocytes from patients with untreated T2DM [37]. Similarly, TLR2 and TLR4 mediate FFA-induced activation of inflammatory pathways and metabolic signaling in insulin resistance, mainly though NF- $\mathrm{KB}$ activation $[38,39]$.

Adipose tissue appears to be a major site of production of inflammatory mediators, as a result of the cross-talk between adipose cells, macrophages, and other infiltrating immune cells. An increased TLR2 expression has been demonstrated in subcutaneous adipose tissue of patients with T2DM [40]. Similarly, an increased TLR4 gene and protein expression was found in the muscle biopsies from obese subjects and patients with T2DM compared to thin subjects, along with an increase in NF- $\kappa B$ signaling and release of IL-6 and TNF- $\alpha$ [41].

The activation of TLR4 induces inflammation in adipocytes in human and murine models of T2DM [42,43]. A recent study showed an increase in TLR-expressing $B$ cells in patients with
T2DM, whereas no changes in TLR expression on monocytes were observed in diabetic patients on treatment with anti-inflammatory medications [44]. Notably, polymorphisms in the TLR3 gene seem to be associated to the risk of T1DM. In fact, rs5743313 and rs117221827 polymorphisms were associated with an early age at diagnosis and a worse glycemic control [45]. While the presence of TLR4 $+3725 \mathrm{G} / \mathrm{C}$ polymorphism seems to be a novel protective factor against T2DM [46], the presence of a nonsense polymorphism (R392X) in TLR5 gene seems to protect from obesity and to predispose to T2DM [47]. However, these results should be investigated in prospective series. Of note, exercise, but not diet-induced weight loss, can modulate the role of TLRs in diabetes $[48,49]$.

Most interestingly, several studies reported correlation between TLR expression and developing of diabetic complications. Experimental studies demonstrated that TLR2 and TLR4 could be important participants in the progression of atherosclerosis in diabetes [50,51].

Furthermore, TLR4 is upregulated in diabetic cardiomyocytes and plays a role in regulating lipid accumulation in cardiac muscle after the onset of type 1 diabetes, which may contribute to cardiac dysfunction [52].

\section{Therapeutic perspectives}

An improved understanding of the mechanisms linking inflammation to diabetes mellitus has stimulated interest in targeting inflammatory pathways as a part of the strategy to prevent or to control diabetes mellitus and its complications. However, the current strategies to decrease inflammation in diabetes are empirical and some of these include statins, which can reduce TLR2 and TLR4 expression [53], PPAR- $\gamma$ agonists, angiotensin receptor blockers (ARBs) and omega-3 fatty acids. In addition, phytochemicals may be also employed, such as D vitamin [54], orange juice [55] and citrus flavonoid naringenin [56].

To date, there are no approved therapeutic agents targeting TLRs for diabetes. Recently, the effects of immune tolerance, induced by chronic administration of TLR2 agonist Pam $3 \mathrm{CSK}_{4}$, and Dipeptidyl peptidase 4 (DPP4) inhibitors have been investigated. Diabetogenic $\mathrm{T}$ cell priming by DCs was attenuated by chronic treatment with Pam3CSK 4 , indicating DC tolerance. The association with DPP4 could achieve normoglycemia by TLR2 tolerization in combination with DPP4 inhibition but not by TLR2 tolerization or DPP4 inhibition alone [57]. In the same view, the use of TLR9 antagonist oligodeoxynucleotide or chloroquine inhibited bone marrow-derived DCs activation and CD8(+) T cell priming in response to $\mathrm{CpG}$, thus delaying the spontaneous onset of diabetes in NOD mice [58].

At present, a phase II trial (NCT01151605) is ongoing to evaluate the suppression of TLRs by insulin in lean, obese and T2DM patients. Another study (NCT01740817) is running to determine whether a lipid infusion can up-regulate TLR4 signaling in human subjects with obesity and/or diabetes and an observational trial (NCT01561664) is evaluating the regulation of inflammation in obese patients by muscle and fat biopsy. Finally, a phase 4 study (NCT01250340) is assessing the role of TLRs in the pathophysiology of T2DM and associated atherosclerosis in patients treated with aspirin or placebo.

In the last years, a major focus has been reserved to the relationship among exercise, inflammation and innate immunity. The expression of TLR2 and TLR4 on human monocytes in vivo is markedly reduced by strenuous exercise [59]. Moreover, it has been reported that down-regulation of TLR2 and TLR4 expression was associated with improved insulin sensitivity after diet-induced weight loss in human subjects with abnormal glucose tolerance and metabolic syndrome. 


\section{Role of TLRs in pancreatic cancer}

Pancreatic Ductal Adenocarcinoma (PDAC), an aggressive cancer, interacts with stromal cells to produce a highly inflammatory tumor microenvironment in order to promotes tumor growth and invasiveness. TLRs mediate interactions between environmental stimuli and innate immunity and trigger proinflammatory signaling cascades. It is likely that TLR activation, either directly or indirectly via stromal cell activation, promotes an aggressive phenotype in the at-risk epithelial cells, which, in turn, induces oncogene-induced senescence (OIS). The ligation of TLRs causes derangements in several tumor suppressor proteins (such as p16, p21, p27, p53 and pRb), STAT3 activation and interfaces with Notch, NF- $\kappa B$ and MAP kinase pathways [60]. These effects seem to be due to stromal cells, since chimeric mice with $\mathrm{Tlr} 4^{-1-}$ or Tlr7 ${ }^{-1-}$ bone marrows, were partially protected from pancreatic carcinogenesis [60,61].

Zambirinis and colleagues explored the effects of TLR-MyD88/TRIF signaling on pancreatic carcinogenesis in p48Cre; LslKras ${ }^{\text {G12D }}$ pancreatic cancer mouse model. Mice treated with either TLR3, TLR4 or TLR7 agonists exhibited a dramatic acceleration of pancreatic cancer progression, characterized by more advanced pre-invasive (PanIN) lesions and a higher number of invasive foci, as well as increased fibrosis and augmented immune infiltrate [62].

The inhibition of MYD88 in DCs leads CD4 ${ }^{+}$T cells toward a $\mathrm{T}_{\mathrm{H}} 2$ profile and to an acceleration of cancer progression by perpetuating inflammation [61]. Similarly, the activation of STAT3 and the upregulation of NOTCH receptors and ligands occur in both the epithelial and the stromal component of PDAC and contribute via NFKB and MAPK to the aggressive tumor phenotype induced by TLR7 activation [63].

Supporting data came from Ochi et al. They revealed the primary role for DCs in pancreatic carcinogenesis and showed that the blockade of TLR4 signaling, via TRIF, is protective against pancreatic cancer. In the same study, they showed that MyD88 inhibition, by augmenting the DC- $\mathrm{T}_{\mathrm{H}} 2$ axis, can exacerbate pancreatic inflammation and neoplastic transformation [61].

LPS-related TLR4 signaling could be a triggering factor in the initiation and progression of pancreatic cancer $[64,65]$, directly modulating the transition from pancreatic inflammation to pancreatic cancer in genetically engineered mouse models $[61,66]$. In PDAC tissue, TLR4 is expressed and its levels have been shown to correlate with tumor size, lymph node involvement, venous invasion, and pathological stage [67]. Moreover, TLR4 expression in pancreatic cancer cells is up-regulated via HIF- $1 \alpha$ in response to hypoxic stress and underscore the crucial role of HIF- $1 \alpha$-induced TLR4 in PDAC tumor growth [68]. Patients with overexpressed TLR4 or overexpressed HIF- $1 \alpha$ had a significantly shorter survival period compared to the patients with normal expression, while longer survival $(p=0.014)$ was reported among patients with neither TLR4 nor HIF-1 $\alpha$ over expression [69].

The epithelial-mesenchymal transition (EMT) in pancreatic cancer promoted by M2-polarized tumor-associated macrophages partially involves the TLR4/IL-10 signaling pathway (Fig. 3). Indeed, the application of TLR4 siRNA and neutralizing antibodies against TLR4 and IL-10 markedly inhibited the reduction of epithelial marker E-cadherin and the upregulation of mesenchymal markers snail and vimentin induced by TLR4 [70].

\section{Therapeutic perspectives}

TLR2 seems to be expressed in over $70 \%$ of pancreatic tumors but not in normal pancreas tissue. The potential use of synthetic TLR2 agonists for the enhancement of cancer immunotherapy is an active area of research. Three mechanisms are involved in the antitumor activity of TLR2: induction of apoptosis in TLR2-positive tumors, enhancement of the innate and T-cell immunity, and improvement of cytotoxic antibody function. Huynh and colleagues demonstrated the PDAC specific retention of the fluorescently labeled compound IRDye800CW-Mpr-, in vivo, using mice bearing TLR2 expressing xenografts [71]. The intraoperative use for increasing the detection of negative resection margins should be prospectively investigated.

A phase I/II trial examined the TLR 2/6 agonist MALP-2 in combination with gemcitabine in patients with locally advanced PDACs. Ten patients were injected intratumorally during surgery with 20-30 mg of MALP-2 followed by postoperative chemotherapy. The median survival was 9.3 months, with two patients still alive after 31 months [72].

Immune responses are impaired in pancreatic cancer patients. A promising strategy for interfering with tumor immune evasion can

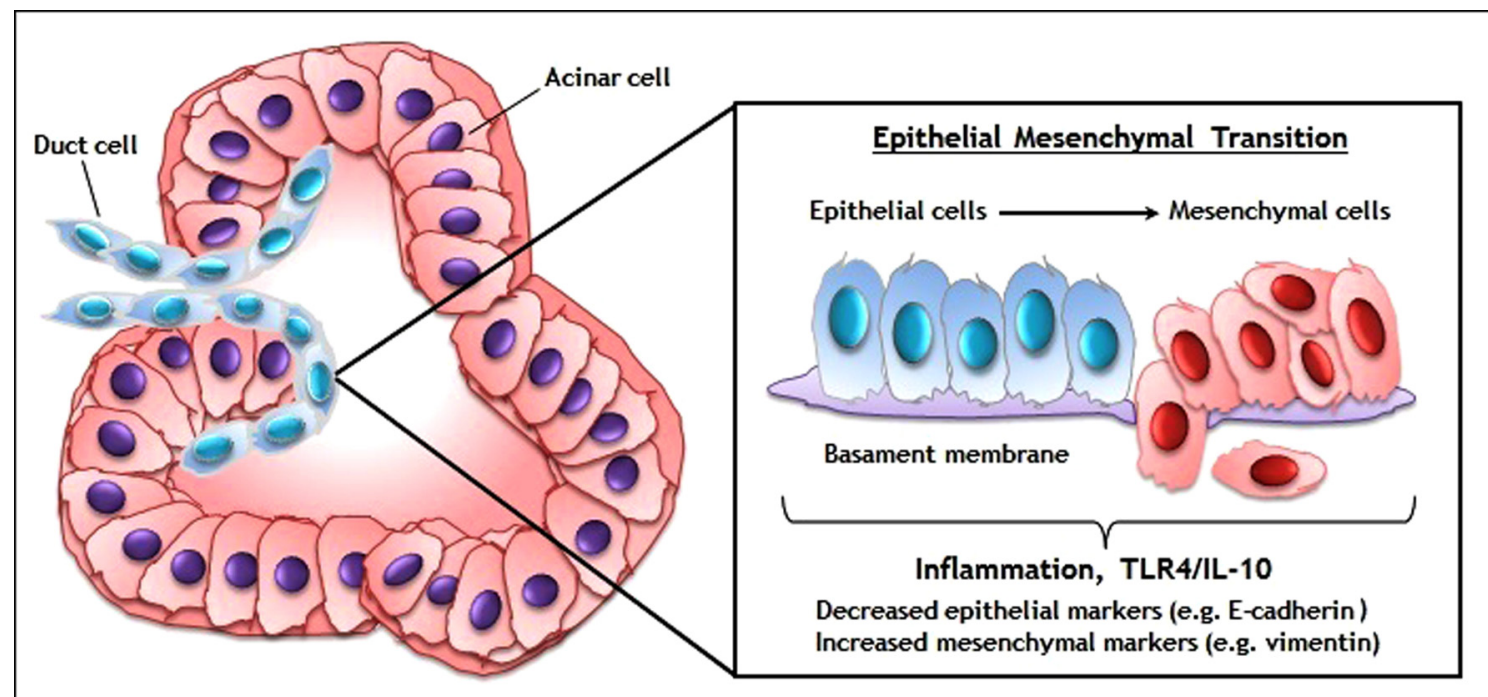

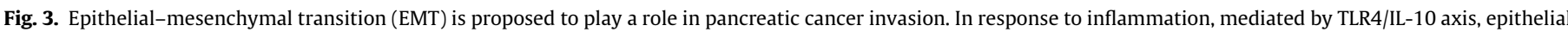

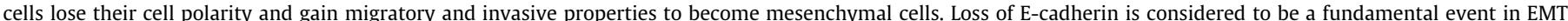

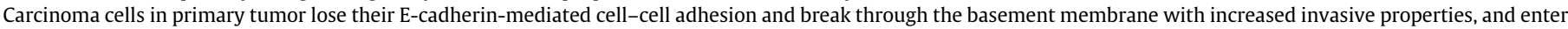
the bloodstream through intravasation. 
be represented by the combination of vaccines based on immune stimulatory complexes (ISCOM) and TLR agonists. Jacobs et al. investigated the efficacy of an ISCOM vaccine alone or in combination with the TLR9 agonist CpG in a murine pancreatic carcinoma model. Unfortunately, ISCOM vaccine, alone, did not affect tumor growth, but its combination with $\mathrm{CpG}$ enhanced cytotoxic $\mathrm{T}$ lymphocyte responses and induced regression of pancreatic tumors in a $\mathrm{CD}^{+} \mathrm{T}$ cell-dependent manner [73].

Other data on the potential role of TLR9 in cancer were showed by Rosa et al. A TLR9 agonist immunomodulatory oligonucleotide (IMO) alone or in combination with cetuximab was investigated in subcutaneous colon and orthotopic pancreatic cancer models harboring K-Ras mutations and resistance to EGFR inhibitors. They reported that IMO markedly inhibited growth of K-Ras mutant colon and pancreatic cancers in vitro and in nude mice and cooperated with cetuximab via multiple mechanisms of action, suggesting for its use in cetuximab-resistant colorectal and pancreatic cancers [74].

Zhou et al. evaluated whether 6-shogaol, a hydroxycinnamic acid derivative extracted from ginger, could suppress pancreatic cancer progression and potentiate response to gemcitabine treatment in vitro and in vivo. They showed that 6-shogaol prevented the activation of TLR4/NF- $\kappa B$ signaling and suppressed key cell survival regulators including cyclooxygenase 2 (COX-2), cyclinD1, survivin, cIAP-1, XIAP, Bcl-2, and MMP-9. In addition, 6-shogaol inhibited the growth of human pancreatic tumors and sensitized them to gemcitabine by suppressing of TLR4/NF- $\kappa B$-mediated inflammatory pathways linked to tumorigenesis [75].

MicroRNAs (miRNAs) are a class of highly conserved non-coding small RNAs. It's been shown that several miRNAs, including miR-301a, can affect the expression of different proteins in the NF- $\kappa B$ pathway in immune cells, thus affecting the production of inflammatory mediators such as IL-1 $\beta$ and TNF $\alpha$. miR-301a is specifically over-expressed in a number of cancers, including PDAC [76].

miR-301a down-regulates its target gene, NF- $\mathrm{B}$ repressing factor (NKRF), by enhancing NF- $\kappa B$ activity. The downregulation of miR-301a expression leads to a suppressed TLR-dependent innate immune response by reducing macrophage COX-2 and IL- 6 expression. These data suggest that miR-301a inhibition or NKRF up-regulation can reduce NF- $\mathrm{KB}$ target gene expression and tumor growth, representing a potential therapeutic approach in patients with PDAC [77].

\section{Conclusions}

Pancreatic inflammation, diabetes and tumor progression involves TLR-mediated irregular and uninhibited production of proinflammatory cytokines, chemokines, and also immunosuppressive cytokines, suggesting that the discovery of TLR antagonists might be an ideal therapeutic strategy. However, TLR antagonists could pose the risk to compromise host immunity, mostly in cancer patients. Although the use of TLR agonists and antagonists may provide a benefit in patients with pancreatic cancer and disease, interest is centered on the potential to complement exist ing modes of therapy with radiation, monoclonal antibodies or cyto toxic drugs.

In summary, TLRs represent promising therapeutic targets in patients with AP, diabetes and PDAC, although further research and elucidation of involved mechanisms are warranted in order to explore their potential future clinical implications.

\section{Founding}

No specific funding was disclosed.

\section{Conflicts of interest}

The authors declared no conflicts of interest.

\section{References}

[1] Kawai T, Akira S. Toll-like receptors and their crosstalk with other innate receptors in infection and immunity. Immunity 2011;34:637-50.

[2] Kawai T, Akira S. The role of pattern-recognition receptors in innate immunity: update on toll-like receptors. Nat Immunol 2010;11:373-84.

[3] Lee CC, Avalos AM, Ploegh HL. Accessory molecules for toll-like receptors and their function. Nat Rev Immunol 2012;12:168-79.

[4] Rittirsch D, Flierl MA, Ward PA. Harmful molecular mechanisms in sepsis. Nat Rev Immunol 2008;8:776-87.

[5] Piccinini AM, Midwood KS. DAMPening inflammation by modulating TLR signalling. Mediators Inflamm 2010;2010:672395

[6] O'Neill LA, Bowie AG. The family of five: TIR-domain containing adaptors in Toll-like receptor signalling. Nat Rev Immunol 2007;7:353-64.

[7] Kawai T, Akira S. TLR signaling. Semin Immunol 2007;19:24-32.

[8] Frossard JL, Steer ML, Pastor CM. Acute pancreatitis. Lancet 2008;371:143-52.

[9] Hoque R, Malik AF, Gorelick F, Mehal WZ. Sterile inflammatory response in acute pancreatitis. Pancreas 2012;41:353-7.

[10] Smolarczyk R, Cichoń T, Jarosz M, Szala S. HMGB1 - its role in tumor progression and anticancer therapy. Postepy Hig Med Dosw 2012;66:913-20.

[11] Ding JL, Li Y, Zhou XY, Wang L, et al. Potential role of the TLR4/IRAK-4 signaling pathway in the pathophysiology of acute pancreatitis in mice. Inflamm Res 2009;58:783-90.

[12] Awla D, Abdulla A, Regnér S, Thorlacius H. TLR4 but not TLR2 regulates inflammation and tissue damage in acute pancreatitis induced by retrograde infusion of taurocholate. Inflamm Res 2011;60:1093-8.

[13] Akashi-Takamura S, Miyake K. TLR accessory molecules. Curr Opin Immunol 2008;20:420-5.

[14] Hoque R, Sohail M, Malik A, et al. TLR9 and the NLRP3 inflammasome link acinar cell death with inflammation in acute pancreatitis. Gastroenterology 2011:141:358-69.

[15] Klein Klouwenberg PM, Ong DS, Bonten MJ, Cremer OL. Classification of sepsis, severe sepsis and septic shock: the impact of minor variations in data capture and definition of SIRS criteria. Intensive Care Med 2012;38:811-9.

[16] Zhai Y, Shen XD, O'Connell R, et al. Cutting edge: TLR4 activation mediates liver ischemia/reperfusion inflammatory response via IFN regulatory factor 3dependent MyD88-independent pathway. J Immunol 2004;173:7115-9.

[17] Matsuda N, Hattori Y. Systemic inflammatory response syndrome (SIRS): molecular pathophysiology and gene therapy. J Pharmacol Sci 2006;101: 189-98.

[18] Bhatia M, Brady M, Shokuhi S, et al. Inflammatory mediators in acute pancreatitis. J Pathol 2000;190:117-25.

[19] Johnson GB, Brunn GJ, Platt JL. Cutting edge: an endogenous pathway to systemic inflammatory response syndrome (SIRS)-like reactions through tolllike receptor 4. J Immunol 2004;172:20-4.

[20] Vaz J, Akbarshahi H, Andersson R. Controversial role of toll-like receptors in acute pancreatitis. World J Gastroenterol 2013;19:616-30.

[21] Liu M, Gu M, Xu D, et al. Protective effects of toll-like receptor 4 inhibitor eritoran on renal ischemia-reperfusion injury. Transplant Proc 2010;42: 1539-44.

[22] Makkouk A, Abdelnoor AM. The potential use of toll-like receptor (TLR) agonists and antagonists as prophylactic and/or therapeutic agents. Immunopharmacol Immunotoxicol 2009;31:331-8.

[23] Rice TW, Wheeler AP, Bernard GR, et al. A randomized, double-blind, placebocontrolled trial of TAK-242 for the treatment of severe sepsis. Crit Care Med 2010;38:1685-94.

[24] Opal SM, Laterre PF, Francois B, et al. Effect of eritoran an antagonist of MD2TLR4, on mortality in patients with severe sepsis: the ACCESS randomized trial. JAMA 2013;309:1154-62.

[25] Sawa H, Ueda T, Takeyama Y, et al. Blockade of high mobility group box-1 protein attenuates experimental severe acute pancreatitis. World J Gastroenterol 2006;12:7666-70.

[26] Guillaumes S, Blanco I, Villanueva A, et al. Chloroquine stabilizes pancreatic lysosomes and improves survival of mice with diet-induced acute pancreatitis. Pancreas 1997; 14:262-6.

[27] Norman J, Franz M, Messina J, et al, Interleukin-1 receptor antagonist decreases severity of experimental acute pancreatitis. Surgery 1995;117: 648-55.

[28] Ding JL, Zhou ZG, Zhou XY, et al. Attenuation of acute pancreatitis by peroxisome proliferator-activated receptor- $\alpha$ in rats: the effect on toll-like receptor signaling pathways. Pancreas 2013;42:114-22.

[29] Shaw JE, Sicree RA, Zimmet PZ. Diabetes atlas-global estimates of the prevalence of diabetes for 2010 and 2030. Diabetes Res Clin Pract 2010;87: $4-14$.

[30] Wild S, Roglic G, Green A, et al. Global prevalence of diabetes: estimates for the year 2000 and projections for 2030. Diabetes Care 2004;27:1047-53.

[31] Devaraj S, Dasu MR, Rockwood J, et al. Increased toll-like receptor (TLR)2 and TLR4 expression in monocytes from patients with type 1 diabetes: further evidence of a proinflammatory state. J Clin Endocrinol Metab 2008;93:578-83. 
[32] Devaraj S, Jialal I. Increased secretion of IP-10 from monocytes under hyperglycemia is via the TLR2 and TLR4 pathway. Cytokine 2009;47:6-10.

[33] Devaraj S, Dasu MR, Park SH, et al. Increased levels of ligands of toll-like receptors 2 and 4 in type 1 diabetes. Diabetologia 2009;52:1665-8.

[34] Kim DH, Lee JC, Kim S, et al. Inhibition of autoimmune diabetes by TLR2 tolerance. J Immunol 2011;187:5211-20.

[35] Devaraj S, Tobias P, Jialal I. Knockout of toll-like receptor-4 attenuates the proinflammatory state of diabetes. Cytokine 2011;55:441-5.

[36] Mraz M, Lacinova Z, Drapalova J, et al. The effect of very-low calorie diet on $m$ RNA expression if inflammation-related genes in subcutaneous adipose tissue and peripheral monocytes of obese patients with type 2 diabetes mellitus. Clin Endocrinol Metab 2011;96:E606-13.

[37] Dasu MR, Devaraj S, Park S, Jialal I. Increased toll-like receptor (TLR) activation and TLR ligands in recently diagnosed type 2 diabetic subjects. Diabetes Care 2010;33:861-8.

[38] Shi H, Kokoeva MV, Inouye K, Tzameli I, Yin H, Flier JS. TLR4 links innate immunity and fatty acid-induced insulin resistance. J Clin Invest 2006;116: 3015-25.

[39] Senn JJ. Toll-like receptor-2 is essential for the development of palmitateinduced insulin resistance in myotubes. J Biol Chem 2006;281:26865-75.

[40] Creely SJ, McTernan PG, Kusminsji CM, et al. Lipopolysaccharide activates an innate immune system response in human adipose tissue in obesity and type 2 diabetes. Am J Physiol Endocrinol Metab 2007;292:E740-7.

[41] Reyna SM, Ghosh S, Tantiwong P, et al. Elevated toll-like receptor 4 expression and signaling in muscle from insulin resistant subjects. Diabetes 2008;57: 2595-602.

[42] Song MJ, Kim KH, Yoon JM, Kim JB. Activation of toll-like receptor 4 is associated with insulin resistance in adipocytes. Biochem Biophys Res Commun 2006;346:739-45.

[43] Vitseva OI, Tanriverdi K, Tchkonia TT, et al. Inducible toll-like receptor and NF-_B regulatory pathway expression in human adipose tissue. Obesity 2008;16:932-7.

[44] Jagannathan M, McDonnell M, Liang Y, et al. Toll-like receptors regulate B cell cytokine production in patients with diabetes. Diabetologia 2010;53:1461-71.

[45] Assmann TS, Brondani LA, Bauer AC, Canani LH, Crispim D. Polymorphisms in the toll-like receptor 3 (TLR3) gene are associated with risk to type 1 diabetes mellitus. Eur J Endocrinol 2014;170:519-27.

[46] Cai H, Cai J, Tao G. Association of toll-like receptor 4 polymorphisms with type 2 diabetes mellitus. APMIS 2013;121:605-11.

[47] Al-Daghri NM, Clerici M, Al-Attas O, et al. A nonsense polymorphism (R392X) in TLR5 protects from obesity but predisposes to diabetes. J Immunol 2013;190:3716-20.

[48] Nickel T, Emslander I, Sisic Z, et al. Modulation of dendritic cells and toll-like receptors by marathon running. Eur J Appl Physiol 2012;112:1699-708.

[49] Lambert CP, Wright NR, Finck BN, Villareal DT. Exercise but not diet-induced weight loss decreases skeletal muscle inflammatory gene expression in frail obese elderly persons. J Appl Physiol 2008;105:473-8.

[50] Li H, Sun B. Toll-like 4 receptor 4 in atherosclerosis. J Cell Mol Med 2007;11:88-95.

[51] Liu X, Ukai T, Yumoto H, et al. Toll-like receptor 2 plays a critical role in the progression of atherosclerosis that is independent of dietary lipids. Atherosclerosis 2008; 196:146-54.

[52] Dong B, Qi D, Yang L, et al. TLR4 regulates cardiac lipid accumulation and diabetic heart disease in the nonobese diabetic mouse model of type 1 diabetes. Am J Physiol Heart Circ Physiol 2012;303:H732-42.

[53] Niessner A, Steiner S, Speidl WS, et al. Simvastatin suppresses endotoxininduced upregulation of toll-like receptors 4 and 2 in vivo. Atherosclerosis 2006;189:408-13.

[54] Devaraj S, Yun JM, Duncan-Staley CR, Jialal I. Low vitamin D levels correlate with the proinflammatory state in type 1 diabetic subjects with and without microvascular complications. Am J Clin Pathol 2011;135:429-33.

[55] Ghanim H, Sia CL, Upadhyay M, et al. Orange juice neutralizes the proinflammatory effect of a high-fat, high-carbohydrate meal and prevents endotoxin increase and toll-like receptor expression. Am J Clin Nutr 2010;91:940-9.
[56] Yoshida H, Watanabe W, Oomagari H, Tsuruta E, Shida M, Kurokawa M. Citrus flavonoid naringenin inhibits TLR2 expression in adipocytes. J Nutr Biochem 2013;24:1276-84.

[57] Lee MS. Treatment of autoimmune diabetes by inhibiting the initial event Immune Network 2013;13:194-8.

[58] Zhang Y, Lee AS, Shameli A, et al. TLR9 blockade inhibits activation of diabetogenic CD8+ $\mathrm{T}$ cells and delays autoimmune diabetes. J Immunol 2010;184:5645-53.

[59] Gleeson M, McFarlin B, Flynn M. Exercise and toll-like receptors. Exercise Immunol Rev 2006;12:34-53.

[60] Ochi A, Graffeo CS, Zambirinis CP, et al. Toll-like receptor 7 regulates pancreatic carcinogenesis in mice and humans. J Clin Invest 2012;122: 4118-29.

[61] Ochi A, Nguyen AH, Bedrosian AS, et al. MyD88 inhibition amplifies dendritic cell capacity to promote pancreatic carcinogenesis via Th2 cells. J Exp Med 2012;209:1671-87.

[62] Zambirinis CP, Ochi A, Barilla R, Greco S, Deutsch M, Miller G. Induction of TRIF- or MYD88-dependent pathways perturbs cell cycle regulation in pancreatic cancer. Cell Cycle 2013;12. 1153115-4.

[63] Zambirinis CP, Miller G. Signaling via MYD88 in the pancreatic tumor microenvironment: a double-edged sword. Oncoimmunology 2013;2: e22567.

[64] Del Pozo JL. Primers on molecular pathways: lipopolysaccharide signaling potential role in pancreatitis and pancreatic cancer. Pancreatology 2010;10: $114-8$.

[65] Mai CW, Kang YB, Pichika MR. Should a toll-like receptor 4 (TLR-4) agonist or antagonist be designed to treat cancer? TLR-4: its expression and effects in the ten most common cancers. Onco Targets Ther 2013;6:1573-87.

[66] Ikebe M, Kitaura Y, Nakamura M, et al. Lipopolysaccharide (LPS) increases the invasive ability of pancreatic cancer cells through the TLR4/MyD88 signaling pathway. J Surg Oncol 2009;100:725-31.

[67] Zhang JJ, Wu HS, Wang L, Tian Y, Zhang JH, Wu HL. Expression and significance of TLR4 and HIF-1alpha in pancreatic ductal adenocarcinoma. World J Gastroenterol 2010;16:2881-8.

[68] Fan P, Zhang JJ, Wang B, et al. Hypoxia-inducible factor-1 up-regulates the expression of toll-like receptor 4 in pancreatic cancer cells under hypoxic conditions. Pancreatology 2012;12:170-8.

[69] Molteni M, Marabella D, Orlandi C, Rossetti C. Melanoma cell lines are responsive in vitro to lipopolysaccharide and express TLR-4. Cancer Lett 2006;235:75-83.

[70] Liu CY, Xu JY, Shi XY, et al. M2-polarized tumor-associated macrophages promoted epithelial-mesenchymal transition in pancreatic cancer cells, partially through TLR4/IL-10 signaling pathway. Lab Invest 2013;93: 844-54.

[71] Huynh AS, Chung WJ, Cho HI, et al. Novel toll-like receptor 2 ligands for targeted pancreatic cancer imaging and immunotherapy. J Med Chem 2012;55:9751-62.

[72] Schmidt J, Welsch T, Jäger D, Mühlradt PF, Büchler MW, Märten A Intratumoural injection of the toll-like receptor-2/6 agonist 'macrophageactivating lipopeptide-2' in patients with pancreatic carcinoma: a phase I/II trial. Br J Cancer 2007;97:598-604.

[73] Jacobs C, Duewell P, Heckelsmiller K, et al. An ISCOM vaccine combined with a TLR9 agonist breaks immune evasion mediated by regulatory $\mathrm{T}$ cells in an orthotopic model of pancreatic carcinoma. Int J Cancer 2011;128:897-907.

[74] Rosa R, Melisi D, Damiano V, et al. Toll-like receptor 9 agonist IMO cooperates with cetuximab in K-ras mutant colorectal and pancreatic cancers. Clin Cancer Res 2011;17:6531-41.

[75] Zhou L, Qi L, Jiang L, et al. Antitumor activity of gemcitabine can be potentiated in pancreatic cancer through modulation of TLR4/NF- $\kappa B$ signaling by 6-shogaol. AAPS J 2014;16:246-57.

[76] Lu Z, Li Y, Takwi A, et al. MiR-301a as an NF-jB activator in pancreatic cancer cells. EMBO J 2011;30:57-67.

[77] Huang L, Liu Y, Wang L, et al. Down-regulation of miR-301a suppresses pro-inflammatory cytokines in toll-like receptor-triggered macrophages. Immunology 2013;140:314-22. 